\title{
The Effect of Different Coherence-Based Beamforming Techniques on the Accuracy of High Frame Rate Speckle Tracking Echocardiography
}

\author{
Marta Orlowska \\ Laboratory of Cardiovascular \\ Imaging and Dynamics, Dept. of \\ Cardiovascular Sciences \\ KU Leuven \\ Leuven, Belgium \\ marta.orlowska@kuleuven.be
}

\author{
Alessandro Ramalli \\ Dept. of Information Engineering \\ University of Florence \\ Florence, Italy \\ alessandro.ramalli@unifi.it
}

Jens-Uwe Voigt

Laboratory of Cardiovascular

Imaging and Dynamics, Dept. of

Cardiovascular Sciences

KU Leuven

Leuven, Belgium

jens-uwe.voigt@uzleuven.be

\author{
Stephanie Bézy \\ Laboratory of Cardiovascular \\ Imaging and Dynamics, Dept. of \\ Cardiovascular Sciences \\ KU Leuven \\ Leuven, Belgium \\ stephanie.bezy@kuleuven.be \\ Jan D'hooge \\ Laboratory of Cardiovascular \\ Imaging and Dynamics, Dept. of \\ Cardiovascular Sciences \\ KU Leuven \\ Leuven, Belgium \\ jan.dhooge@uzleuven.be
}

\author{
Giulia Matrone \\ Dept. of Electrical, Computer and \\ Biomedical Engineering \\ University of Pavia \\ Pavia, Italy \\ giulia.matrone@unipv.it
}

\begin{abstract}
Although high frame rate (HFR) imaging could be a promising technique for a better assessment of myocardial function, the higher temporal resolution comes with decreased image quality, which affects the accuracy of speckle tracking echocardiography (STE). Since several adaptive beamforming algorithms have already been proposed to improve image quality, the aim of this study was to assess to what extent this impacts the accuracy of HFR STE. Simulated datasets were used to compare 7 coherence-based beamforming schemes. The STE results were similar for different beamformers with root mean square errors between 0.36 and $0.50 \%$. Next, in vivo apical 4chamber views were recorded from 3 healthy volunteers using the same beamforming schemes. The related strain curves were similar for the different beamforming methods and the endsystolic values showed no significant difference among beamformers and volunteers. In conclusion, our findings suggest that the choice of beamforming method does not significantly affect HFR STE in a clinical setting.
\end{abstract}

Keywords-high frame rate, coherence beamforming, speckle tracking, echocardiography

\section{INTRODUCTION}

High frame rate (HFR) imaging is a promising technique for a better assessment of myocardial function. To keep the field of view and increase the temporal resolution, two groups of HFR approaches have been proposed: one has been based on the simultaneous transmission into different directions of multiple focused beams [1], while the second one has been based on defocused waves, i.e. diverging or plane waves, along with massive parallel beamforming and coherent compounding [2], [3]. However, the higher temporal resolution comes at the cost of image quality. To improve image quality, several adaptive beamforming algorithms have

M. Orlowska was supported by FWO grants No G002617N and G092318N. JU. Voigt holds a personal FWO research mandate 1832917N.

A. Ramalli was partially supported by the European Union's Horizon 2020 research and innovation programme under the Marie Skłodowska-Curie grant agreement No 786027 (ACOUSTIC project). S. Bézy was supported by FWO grant No G092318N. already been proposed. For example, the coherence-based beamforming methods exploit the spatial coherence between echo signals detected by individual array elements in order to increase spatial resolution and image contrast compared to the conventional delay-and-sum (DAS) beamforming [4]. However, the different beamformers are usually compared based on the image appearance, but not on the possible impact that they may have on successive further analyses. In [5] it was shown that, qualitatively, B-mode based speckle tracking echocardiography benefited from coherence beamforming methods, despite the subjective and thus observer-dependent nature of those findings. To date, the impact of these beamformers on speckle tracking echocardiography (STE) using radiofrequency (RF) data, where the chosen dynamic range and gain have no influence, has not been studied in detail.

The aim of this study was therefore to quantitatively assess to what extent adaptive beamforming impacts the accuracy of HFR STE. Specifically, seven different beamformers were applied on simulated data and then challenged in recordings made in vivo.

\section{BEAMFORMING TECHNIQUES}

\section{A. Coherence Factor (CF)}

The CF [6] is the ratio of the coherent and incoherent sum of the RF signals $s_{n}(t)$ received by the $n$-th element out of the $\mathrm{N}$ active ones in the aperture in reception after applying dynamic focusing delays:

$$
C F(t)=\frac{\left|\sum_{n=1}^{N} s_{n}(t)\right|^{2}}{N \sum_{n=1}^{N} s_{n}^{2}(t)}
$$

The CF-weighted final image is obtained as: 


$$
y_{C F}(t)=C F(t) y(t)
$$

where $y(t)$ is the output of DAS beamformer:

$$
y(t)=\sum_{n=1}^{N} s_{n}(t)
$$

\section{B. Generalized Coherence Factor (GCF)}

The GCF [6] is a generalization of the CF. It is defined as the ratio of the energy within a given low-frequency component (lower than a cutoff frequency $M_{0}$ ) to the total energy:

$$
G C F(t)=\frac{\sum_{k=0}^{M_{0}}\left|S_{n}(k)\right|^{2}}{\sum_{k=0}^{N-1}\left|S_{n}(k)\right|^{2}}
$$

where $S_{n}(k)$ is the spectrum of $s_{n}(t)$ and $k$ represents the spatial frequency index. The final image is obtained by weighting the DAS image with the GCF.

\section{Phase Coherence (PCF) and Sign Coherence Factor (SCF)}

The PCF [7] is a sample-wise weighting for DAS:

$$
P C F(t)=\max \left\{0.1-\frac{\gamma}{\sigma_{0}} s f(t)\right\}
$$

where $\sigma_{0}=\pi / \sqrt{3}$ is the standard deviation of a uniform distribution between $-\pi$ and $\pi$. $\gamma$ is a sensitivity parameter ranging between 0 and $1 . s f(t)$ is an estimate of the standard deviation of the time-domain phases of $s_{n}(t)$, defined as:

$$
s f(t)=\min \left\{\sigma\left(\varphi_{n}(t)\right), \sigma\left(\varphi_{n}^{A}(t)\right)\right\}
$$

where $\sigma\left(\varphi_{n}(t)\right)$ is the standard deviation of the instantaneous phase of signals $s_{n}(t)$ and $\sigma\left(\varphi_{n}^{A}(t)\right)$ is a set of auxiliary phases to avoid the phase wrapping discontinuity.

The SCF [7] is a simplified version of the PCF, where we consider the distribution of signs instead of the coherence of phases of received signals. The SCF is defined as:

$$
S C F(t)=\left|1-\sigma\left(b_{n}(t)\right)\right|^{\gamma}
$$

where $\sigma\left(b_{n}(t)\right)$ is the standard deviation of the sign of $s_{n}(t)$ and $\gamma$ is a sensitivity parameter.

\section{Short-Lag Filtered-Delay Multiply and Sum Beamforming (SL-FDMAS)}

The SL-FDMAS [8] beamforming is based on the spatial autocorrelation function of the aperture in reception, which is limited to a certain maximum lag (ML) in the computation. It is defined as follow:

$$
\begin{aligned}
y_{S L-D M A S}(t)= & \sum_{L=1}^{M L} \sum_{n=1}^{N-L} \operatorname{sign}\left(s_{n}(t) s_{n+L}(t)\right) * \\
& \sqrt{\left|s_{n}(t) s_{n+L}(t)\right|}
\end{aligned}
$$

\section{E. Nonlinear Beamforming with p-th Root Compression ( $P$ - $D A S)$}

The P-DAS [9] is a nonlinear beamformer based on $\mathrm{p}^{\text {th }}$ root compression, where signal amplitudes are compressed using the signed $\mathrm{p}^{\text {th }}$ root and then restored to the original dimensionality by applying the signed p-power, as follows:

$$
\begin{gathered}
y_{p-D A S}(t)=\operatorname{sign}\left\{\sum_{n=1}^{N} \operatorname{sign}\left(s_{n}(t)\right) \sqrt[p]{\left|s_{n}(t)\right|}\right\} * \\
\left|\sum_{n=1}^{N} \operatorname{sign}\left(s_{n}(t)\right) \sqrt[p]{\left|s_{n}(t)\right|}\right|^{p}
\end{gathered}
$$

where $p$ is a user-defined parameter that tunes the contrast/resolution performance of the algorithm.

\section{METHODS}

\section{A. Imaging settings}

Both for simulations and in-vivo experiments, a $90^{\circ}$-wide sector was imaged at a HFR of $833 \mathrm{~Hz}$ by using a sequence of 6 Diverging Waves (DW) transmitted from a sub-aperture (equal to $1 / 3$ of the probe size) sliding over the total array [10]. The channel data were captured, and images were reconstructed offline using a compounding scheme.

Seven beamformers were compared: DAS, SL-FDMAS, CF, GCF, PCF, SCF and P-DAS. For each beamformer, various values of their design parameters were considered (see TABLE I).

\section{B. STE Accuracy Estimation}

The effect of HFR imaging and different beamforming schemes on the accuracy of STE was assessed on simulated data. A 64-element phased array, with a $60 \%$ bandwidth at $3.5 \mathrm{MHz}$ and a $0.22 \mathrm{~mm}$ pitch, was setup in Field II [11], [12]; then, the raw echo data received from 5 electromechanical models of the heart ( 1 healthy and 4 diseased) [13] were simulated. Moreover, to test the impact of noise on the accuracy, white Gaussian noise was added to the prebeamformed data considering a signal-to-noise ratio (SNR) of $10 \mathrm{~dB}$.

On the reconstructed images, the position of the cardiac wall was automatically extracted and then tracked by a custom-made 2D HFR STE algorithm [14], based on crosscorrelation. The estimation accuracy was computed as the average temporal root-mean-square error (RMSE) between the ground truth $\left(G T_{i}\right)$ and the estimated $\left(E S T_{i}\right)$ Global Longitudinal Strain (GLS) curves, as follows:

$$
R M S E=\sqrt{\sum_{i=1}^{F} \frac{\left(G T_{i}-E S T_{i}\right)^{2}}{F}}
$$

where $i$ is the time index and $\mathrm{F}$ the number of frames.

\section{In Vivo Setup}

Finally, in vivo apical 4-chamber views, from 3 healthy volunteers, were recorded with the ULA-OP 256 scanner [15], connected to a PA230 probe (Easote S.p.A., Florence, Italy), which has 128 elements (pitch $=170 \mu \mathrm{m}$ ) and a central frequency of $2 \mathrm{MHz}$. The myocardial contour was placed on the reconstructed images by an expert cardiologist and tracked 
TABLE I AVERAGE RMSES OBTAINED FOR DIFFERENT BEAMFORMERS. THE DESIGN PARAMETER WITH THE LOWEST RMSE (GREEN) WAS USED FOR IN VIVO SCANS.

\begin{tabular}{|c|c|c|c|c|}
\hline \multirow{3}{*}{$\begin{array}{c}\text { Beamformer } \\
\text { DAS }\end{array}$} & \multirow{2}{*}{\multicolumn{2}{|c|}{ Design parameter }} & \multicolumn{2}{|c|}{ RMSE [\%] } \\
\hline & & & No noise & Noise \\
\hline & - & - & 0.42 & 0.48 \\
\hline $\mathrm{CF}$ & - & - & 0.37 & 0.39 \\
\hline \multirow{5}{*}{ GCF } & \multirow{5}{*}{$\begin{array}{c}\text { Cut-off } \\
\text { frequency } \\
\left(\mathrm{M}_{0}\right)\end{array}$} & 1 & 0.44 & 0.47 \\
\hline & & 2 & 0.46 & 0.49 \\
\hline & & 3 & 0.47 & 0.50 \\
\hline & & 4 & 0.46 & 0.50 \\
\hline & & 5 & 0.45 & 0.49 \\
\hline \multirow{5}{*}{ PCF } & \multirow{5}{*}{ Sensitivity $(\gamma)$} & 0.2 & 0.41 & 0.47 \\
\hline & & 0.4 & 0.40 & 0.46 \\
\hline & & 0.6 & 0.39 & 0.44 \\
\hline & & 0.8 & 0.37 & 0.41 \\
\hline & & 1 & 0.36 & 0.38 \\
\hline \multirow{5}{*}{ SCF } & \multirow{5}{*}{ Sensitivity $(\gamma)$} & 0.2 & 0.38 & 0.43 \\
\hline & & 0.4 & 0.37 & 0.40 \\
\hline & & 0.6 & 0.37 & 0.39 \\
\hline & & 0.8 & 0.38 & 0.40 \\
\hline & & 1 & 0.39 & 0.40 \\
\hline \multirow{2}{*}{ P-DAS } & \multirow{2}{*}{$\begin{array}{l}\text { Power/root } \\
\text { coeff (p) }\end{array}$} & 3 & 0.36 & 0.37 \\
\hline & & 4 & 0.36 & 0.37 \\
\hline \multirow{3}{*}{ SL-FDMAS } & \multirow{3}{*}{$\begin{array}{l}\text { Maximum lag } \\
(\mathrm{ML} /(\mathrm{N}-1))\end{array}$} & 0.16 & 0.43 & 0.49 \\
\hline & & 0.5 & 0.39 & 0.43 \\
\hline & & 1 & 0.36 & 0.38 \\
\hline
\end{tabular}

throughout the cycle using the same STE algorithm [14]. GLS curves and their end-systolic (ES) values were extracted and compared.

\section{RESULTS AND DISSCUSION}

\section{A. STE Accuracy}

The RMSE values obtained in simulations and the design parameter for each beamformer are shown in TABLE I. Calculated RMSEs were similar, i.e. RMSE was low (0.36$0.50 \%)$ compared to the maximum GLS value (9\%). The difference in the obtained error from the images without and with the noise was very small for all the approaches, ranging between 0.01 and $0.06 \%$. Tissue tracking on the DAS dataset gave a RMSE of $0.42 \%$ and $0.48 \%$ on average for images without and with noise, respectively. The $\mathrm{CF}$ approach showed slight improvements $(0.37 \%$ and $0.39 \%$ without and with noise, respectively). For the GCF beamformer, the error was the lowest when the cut-off frequency was set to 1 (on average $0.46 \%$ ). However, the error was similar across different $\mathrm{M}_{0}$ values, ranging from 0.46 to $0.49 \%$ on average. The same behavior was seen with PCF, where the difference in error for different sensitivity parameter settings was small (0.37-0.44\% on average). Nevertheless, the RMSE was the lowest when sensitivity was equal to 1 . SCF datasets obtained the best tracking performance when sensitivity was set to 0.6, giving RMSE of $0.38 \%$ on average. The two tested values for the parameter $\mathrm{p}$ in $\mathrm{p}$-DAS did not impact the RMSE, which was $0.36 \%$ for data without noise and $0.37 \%$ when SNR was equal to $10 \mathrm{~dB}$. Finally, the data beamformed using the SLFDMAS scheme obtained decreasing errors from $\sim 0.46 \%$, when the ratio between the maximum lag and the number of

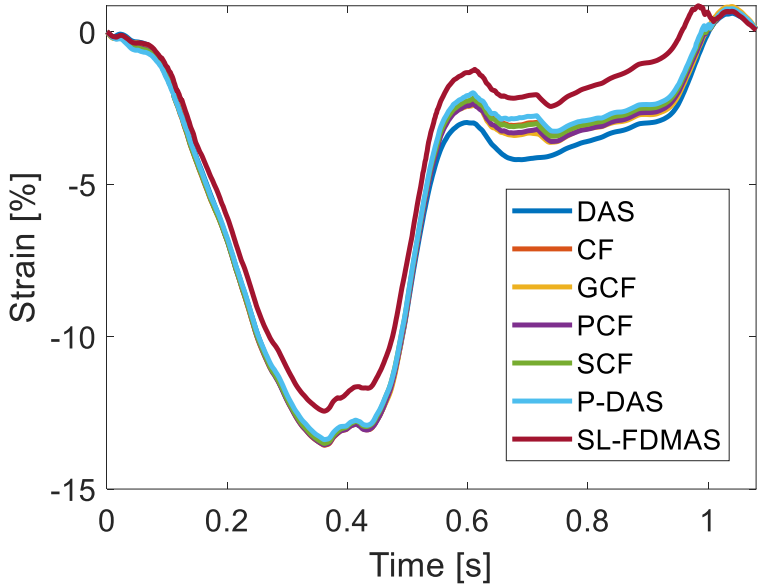

Fig. 1 Example of obtained global longitudinal strain curves.

elements - i.e. $\mathrm{ML} /(\mathrm{N}-1)$ - was set to 0.16 , to $\sim 0.37 \%$ for this ratio equal to 1.

\section{B. In Vivo Cardiac Images}

For each beamformer, the value of the design parameter showing the lowest RMSE in simulations (cf. TABLE I) was chosen as optimal and applied to the in vivo recordings. Fig. 1 shows an example of the obtained GLS curves from the same volunteer for different beamforming schemes. It can be observed that the shape of the curves is very similar. Only the curve associated to SL-FDMAS is slightly different. Indeed, the end-systolic values estimated from different volunteers (see TABLE II) showed no significant differences among different approaches. On average, the values ranged from $14.5 \%$ to $-14.9 \%$ with an exception for SL-FDMAS, for which the average ES value was slightly lower (-13.9\%).

\section{CONCLUSIONS}

In this study, the effect of different coherence-based beamforming techniques on the accuracy of HFR STE was tested. Seven different beamformers were compared and challenged on simulated and in vivo data. Our findings suggest that the choice of the beamforming method does not significantly influence HFR STE in a clinical setting.

\section{REFERENCES}

[1] R. Mallart and M. Fink, "Improved imaging rate through simultaneous transmission of several ultrasound beams," in New Developments in Ultrasonic Transducers and Transducer Systems, Nov. 1992, vol. 1733, pp. 120-131, doi: 10.1117/12.130591.

TABLE II END-SYSTOLIC VALUES OF DIFFERENT BEAMFORMERS AND VOLUNTEERS.

\begin{tabular}{|c|c|c|c|c|}
\hline ES [\%] & Vol 1 & Vol 2 & Vol 3 & Avg \\
\hline DAS & -15.5 & -13.5 & -15.0 & -14.7 \\
\hline CF & -15.5 & -13.6 & -15.3 & -14.8 \\
\hline GCF & -15.4 & -13.4 & -14.8 & -14.5 \\
\hline PCF & -15.6 & -13.5 & -15.5 & -14.9 \\
\hline SCF & -15.5 & -13.5 & -15.4 & -14.8 \\
\hline P-DAS & -15.6 & -13.4 & -15.2 & -14.7 \\
\hline SL-FDMAS & -15.2 & -12.4 & -14.0 & -13.9 \\
\hline
\end{tabular}


[2] H. Hasegawa and H. Kanai, "High-frame-rate echocardiography using diverging transmit beams and parallel receive beamforming," J. Med. Ultrason., vol. 38, no. 3, pp. 129-140, Jul. 2011, doi: 10.1007/s10396-0110304-0.

[3] B. Denarie et al., "Coherent plane wave compounding for very high frame rate ultrasonography of rapidly moving targets," IEEE Trans. Med. Imaging, vol. 32, no. 7, pp. 1265-1276, Jul. 2013, doi: 10.1109/TMI.2013.2255310.

[4] G. Matrone, A. Ramalli, J. D'hooge, P. Tortoli, and G. Magenes, "A Comparison of Coherence-Based Beamforming Techniques in HighFrame-Rate Ultrasound Imaging With Multi-Line Transmission," IEEE Trans. Ultrason. Ferroelectr. Freq. Control, vol. 67, no. 2, pp. 329-340, 2020, doi: 10.1109/TUFFC.2019.2945365

[5] P. Santos et al., "Evaluation of Coherence-Based Beamforming for B-Mode and Speckle Tracking Echocardiography," in 2018 IEEE International Ultrasonics Symposium (IUS), Oct. 2018, pp. 1-4, doi: 10.1109/ULTSYM.2018.8579972.

[6] Pai-Chi Li and Meng-Lin Li, "Adaptive imaging using the generalized coherence factor," IEEE Trans. Ultrason. Ferroelectr. Freq. Control, vol. 50, no. 2, pp. 128-141, Feb. 2003, doi: 10.1109/TUFFC.2003.1182117.

[7] J. Camacho, M. Parrilla, and C. Fritsch, "Phase Coherence Imaging," IEEE Trans. Ultrason. Ferroelectr. Freq. Control, vol. 56, no. 5, pp. 958-974, May 2009, doi: 10.1109/TUFFC.2009.1128.

[8] G. Matrone and A. Ramalli, "Spatial Coherence of Backscattered Signals in Multi-Line Transmit Ultrasound Imaging and Its Effect on ShortLag Filtered-Delay Multiply and Sum Beamforming," Appl. Sci., vol. 8, no. 4, p. 486, Apr. 2018, doi: 10.3390/app8040486
[9] M. Polichetti, F. Varray, J.-C. Béra, C. Cachard, and B. Nicolas, "A Nonlinear Beamformer Based on p-th Root Compression-Application to Plane Wave Ultrasound Imaging," Appl. Sci., vol. 8, no. 4, Art. no. 4, Apr. 2018, doi: 10.3390/app8040599.

[10] V. Komini, P. Santos, and J. D'hooge, "Diverging wave compounding: Direct comparison of two popular approaches," in 2017 IEEE International Ultrasonics Symposium (IUS), Sep. 2017, pp. 1-4, doi: 10.1109/ULTSYM.2017.8092802.

[11] J. A. Jensen, "FIELD: A Program for Simulating Ultrasound Systems," in 10th Nordicbaltic Conference on Biomedical Imaging, Vol. 4, Supplement 1, Part 1:351-353, 1996, pp. 351-353.

[12] J. A. Jensen and N. B. Svendsen, "Calculation of pressure fields from arbitrarily shaped, apodized, and excited ultrasound transducers," IEEE Trans. Ultrason. Ferroelectr. Freq. Control, vol. 39, no. 2, pp. 262-267, Mar. 1992, doi: 10.1109/58.139123.

[13] M. Alessandrini et al., "A Pipeline for the Generation of Realistic 3D Synthetic Echocardiographic Sequences: Methodology and Open-Access Database," IEEE Trans. Med. Imaging, vol. 34, no. 7, pp. 1436-1451, Jul. 2015, doi: 10.1109/TMI.2015.2396632.

[14] M. Orlowska et al., "A novel 2D speckle tracking method for high frame rate echocardiography," IEEE Trans. Ultrason. Ferroelectr. Freq. Control, pp. 1-1, 2020, doi: 10.1109/TUFFC.2020.2985451.

[15] E. Boni et al., "Architecture of an Ultrasound System for Continuous Real-Time High Frame Rate Imaging," IEEE Trans. Ultrason. Ferroelectr. Freq. Control, vol. 64, no. 9, pp. 1276-1284, Sep. 2017, doi: 10.1109/TUFFC.2017.2727980. 